\title{
CALIBRATION EN MAGNITUDES ABSOLUES DE LA CLASSIFICATION BCD. APPLICATION À LA DÉTERMINATION DU MODULE DE DISTANCE DU GRAND NUAGE DE MAGELLAN
}

\author{
L. DIVAN \\ Institut d'Astrophysique, Paris, Observatoire de Haute-Provence, \\ European Southern Observatory, La Silla
}

\begin{abstract}
The calibration of the BCD stellar classification in absolute magnitudes (classification in three parameters $\lambda_{1}, D, \Phi_{b}$ ) was used to determine the distance modulus of the Large Magellanic Cloud. The described method makes use of the spectrophotometric parameters of B and A supergiants of the Large Cloud and gives a value of the distance modulus which is independent from other determinations, in particular from those which are based on the RR Lyrae and the Cepheid variables. The value found for the distance is slightly smaller than those generally admitted. The results are still based only on a limited number of measurements and new observations are in process; however, it seems doubtful that one will obtain much larger values. In other respects, the observations have shown that the parameter $\lambda_{1}$ was still sensitive to luminosity for the B and A stars brighter than $M=-8$ and that the calibration of the $\lambda_{1} D$ diagram in absolute magnitudes can be extended up to $M=-9$.
\end{abstract}

Résumé. La calibration en magnitudes absolues de la classification stellaire BCD (classification à trois paramètres $\lambda_{1}, D, \Phi_{b}$ ) a été utilisée pour déterminer le module de distance du Grand Nuage de Magellan. La méthode qui est décrite fait intervenir les paramètres spectrophotométriques de supergéantes B et A du Grand Nuage et donne une valeur du module de distance indépendante des autres déterminations, en particulier de celles qui sont basées sur les variables RR Lyrae et les Céphéìdes. La valeur trouvée pour la distance est un peu plus faible que celles généralement admises. Elle ne repose encore que sur un petit nombre de mesures et de nouvelles observations sont en cours; il semble cependant douteux que l'on arrive à des valeurs beaucoup plus grandes. Par ailleurs, les observations ont montré que le paramètre $\lambda_{1}$ était encore sensible à la luminosité pour des étoiles $\mathrm{B}$ et A plus brillantes que $M=-8$ et la calibration du diagramme $\lambda_{1} D$ en magnitudes absolues peut être prolongée jusqu'à $M=-9$.

\section{Introduction, calibration de la classification BCD en types spectraux, gradients et magnitudes absolues}

Les méthodes spectrophotométriques décrites dans quatre articles (Baillet et al., 1952; Laffineur, 1952; Chalonge et Servigne, 1952; Chalonge et Divan, 1952) ont permis d'établir une classification stellaire à trois paramètres mesurables: $\lambda_{1}, D$ et $\Phi_{b}$. Plusieurs applications de cette classification ont été décrites, en particulier par Chalonge $(1958,1966)$. Les méthodes et instruments ont été progressivement perfectionnés et l'étude homogène de plusieurs centaines d'étoiles a permis d'obtenir un certain nombre de résultats. Ces résultats, déjà présentés à l'Assemblée Générale de Brighton en Septembre 1970, sont actuellement en cours de publication (Chalonge et Divan, 1973). Dans ce qui suit, nous résumons quelques points importants qui serviront à interprêter les mesures faites sur les étoiles supergéantes du Grand Nuage de Magellan. 


\section{A. CALIBRATION DU Diagramme $\lambda_{1} D$ EN TYPES SPECTRAUX}

Dans le diagramme $\lambda_{1} D$, les courbes séparant les différents types spectraux et classes de luminosité ont été améliorées, principalement dans la région des étoiles supergéantes; la Figure 1 reproduit les nouvelles courbes.

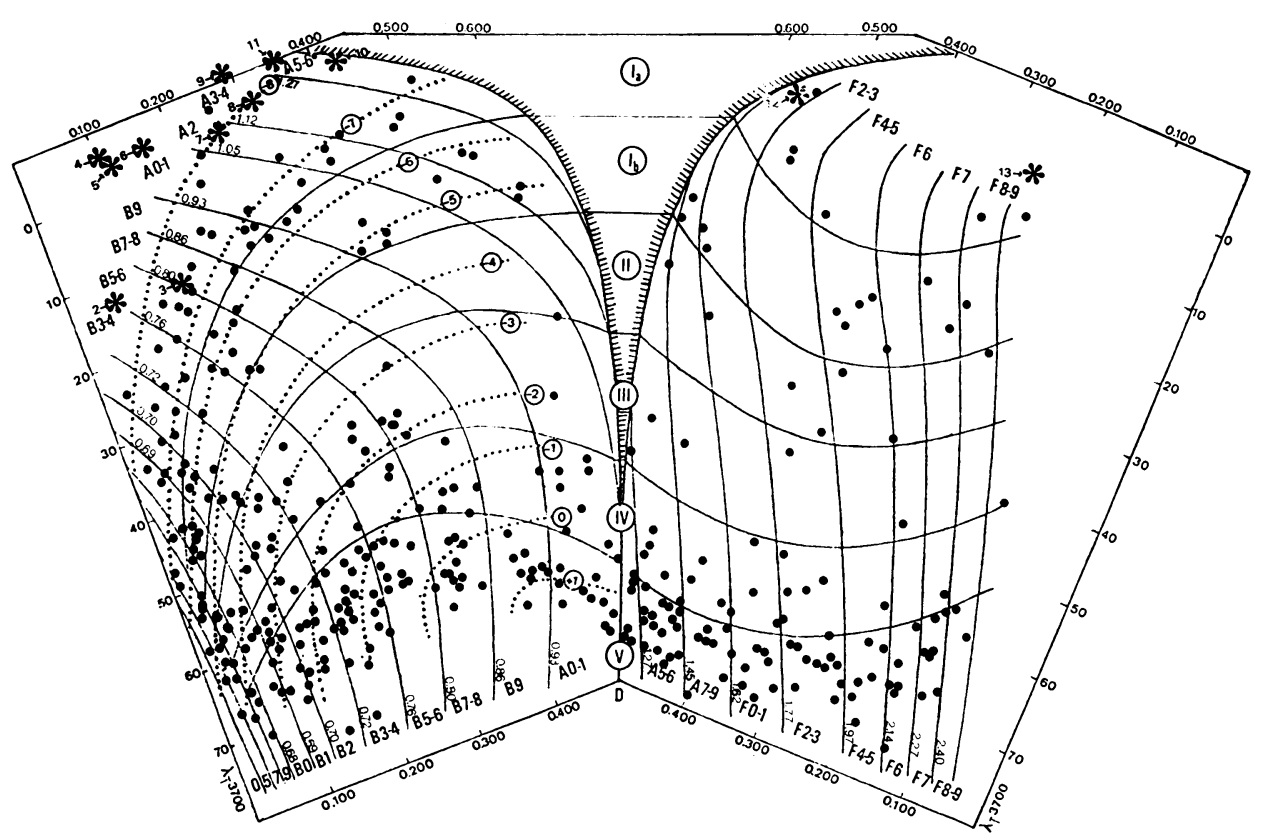

Fig. 1. Diagramme $\lambda_{1} D . \bigcirc$ étoiles galactiques; $*$ étoiles du Grand Nuage de Magellan; - lignes d'égal type spectral et lignes d'égale classe de luminosité (classification MKK); ... lignes d'égale magnitude absolue.

\section{B. Calibration DU Diagramme $\lambda_{1} D$ EN GRADIENTS}

Avec une marge possible d'incertitude de \pm 0.10 les courbes d'égal type spectral sont également des courbes d'égal gradient bleu (domaine spectral 4600-4000 $\AA$ ); la valeur de ce gradient est inscrite sur chacune des courbes dans la Figure 1.

\section{CORRECTIONS D'ABSORPTION INTERSTELLAIRE}

Grâce à la calibration précédente, la connaissance de $\lambda_{1} D$ pour une étoile permet d'obtenir la valeur de son gradient intrinsèque (celui que l'on observerait en l'absence de matière interstellaire) $\Phi_{o b}$. La valeur observée $\Phi_{b}$ donne alors l'excès de gradient $e_{b}=\Phi_{b}-\Phi_{o b}$, excès dont on peut déduire l'absorption $A_{v}$ pour la magnitude $V$ si l'on connaît la forme de la loi d'absorption interstellaire. Avec une loi d'absorption normale, $A_{v}=1.9 e_{b}$.

D. CALIBRATION DU Diagramme $\lambda_{1} D$ EN MAGNitudes ABSOlUES

La mesure de $\lambda_{1} D \Phi_{b}$ pour des étoiles naines et supergéantes situées dans quelques 
amas et associations (Ori I, Pléiades. Per II, Per III, Per I) dont les distances photométriques sont considérées comme bien connues, a permis de placer ces étoiles dans le diagramme $\lambda_{1} D$, puis de calculer pour chacune d'elles la valeur de $A_{v}$ par la formule $A_{v}=1.9 e_{b}$; on en déduit alors $V_{o}$ puis, à partir du module de distance photométrique

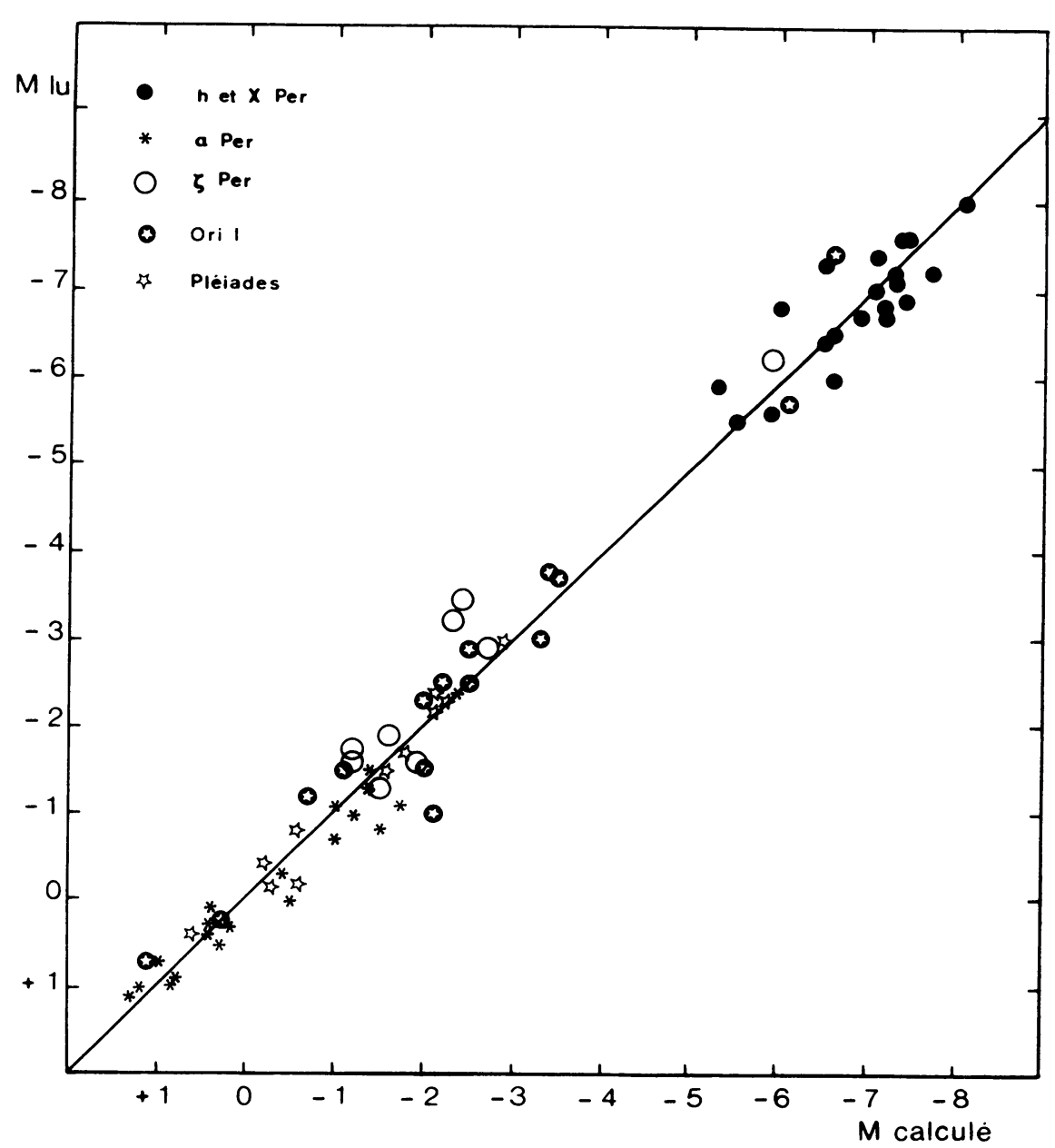

Fig. 2. Comparaison entre les valeurs calculées pour les magnitudes absolues et celles lues sur le réseau des courbes de la Figure 1, pour les étoiles de cinq amas ou associations.

de l'amas, la magnitude absolue $M$. Les magnitudes absolues ainsi obtenues ont été inscrites aux points correspondants du diagramme $\lambda_{1} D$ et il a été possible de tracer un réseau régulier de courbes d'égale magnitude absolue, de $M=+1$ à $M=-8$ (Figure 1), avec un écart moyen entre les valeurs précédemment calculées de $M$ et celles lues sur le réseau inférieur à 0.5 mag. (voir Figure 2). 


\section{Etude de quelques supergéantes du Grand Nuage de Magellan (GNM)}

\section{A. GÉNÉRALITÉS}

L'étude dans le système BCD des supergéantes du GNM est intéressante à plusieurs points de vue:

(i) le paramètre $\lambda_{1}$ très sensible à la luminosité pour les étoiles galactiques les plus brillantes $(M \sim-8)$ permet encore de classer les supergéantes du GNM pour lesquelles les magnitudes absolues atteignent -9 et même -10 .

(ii) la détermination des paramètres $\lambda_{1} D \Phi_{b}$ pour des étoiles du GNM moins lumineuses et se situant en $\lambda_{1} D$ dans les limites de la calibration en magnitudes absolues décrite plus haut, permet d'obtenir une valeur de module de distance du GNM indépendante de celles obtenues par l'intermédiaire des variables RR Lyr ou des Céphéïdes.

\section{B. LES OBSERVATIONS}

Elles ont été faites en Janvier 1971 au télescope de $152 \mathrm{~cm}$ de l'ESO à La Silla, avec l'équipement spectrophotométrique de l'institut d'Astrophysique de Paris (spectrographe Chalonge à chassis oscillant ... etc) suivant les techniques exposées par Chalonge et Divan (1973). En particulier l'absorption atmosphérique et la quantité d'ozone étaient déterminées pour chaque nuit d'observation.

Les quantités $\lambda_{1} D \Phi_{b}$ ont été obtenues pour 13 supergéantes de types $\mathrm{B}, \mathrm{A}$ et $\mathrm{F}$ choisies

(i) parmi les plus lumineuses

(ii) dans un groupe d'étoiles plus faibles que les précédentes de 1 à 1.5 magnitude.

Les résultats sont donnés dans le Tableau I. Les valeurs de $V, B-V, U-B$ sont de Ardeberg et al. (1972).

$\Phi_{r b}, \Phi_{b}$ et $\Phi_{u v}$ sont les gradients moyens obtenus pour les domains spectraux 6200 $4000 \AA, 4600-4000 \AA$ et $3700-3130 \AA$.

Les points représentatifs des étoiles du Tableau I sont portés sur la Figure 1, sauf pour R 81 dont le continu de Balmer est en émission; cette émission modifie les valeurs de $D$ et $\lambda_{1}$ et enlève toute signification à la position du point correspondant dans le diagramme $\lambda_{1} D$.

\section{LES RESULTATS}

\section{(i) Sensibilité du paramètre $\lambda_{1}$ aux grandes luminosités}

L'examen du Tableau I et de la Figure 1 montre une bonne corrélation entre la position des étoiles du GNM par rapport à la courbe $M=-8$ et leur magnitude apparente: les étoiles les plus brillantes sont au-dessus de cette courbe, les moins brillantes audessous. Ceci sera précisé plus loin lorsque les corrections d'absorption interstellaire seront apportées aux magnitudes apparentes considérées ici, mais on peut déjà dire que le paramètre $\lambda_{1}$ est encore sensible à la luminosité pour des étoiles nettement plus lumineuses que les supergéantes galactiques. La classification $\lambda_{1} D$ des étoiles du GNM a donc une signification même pour les plus brillantes. 


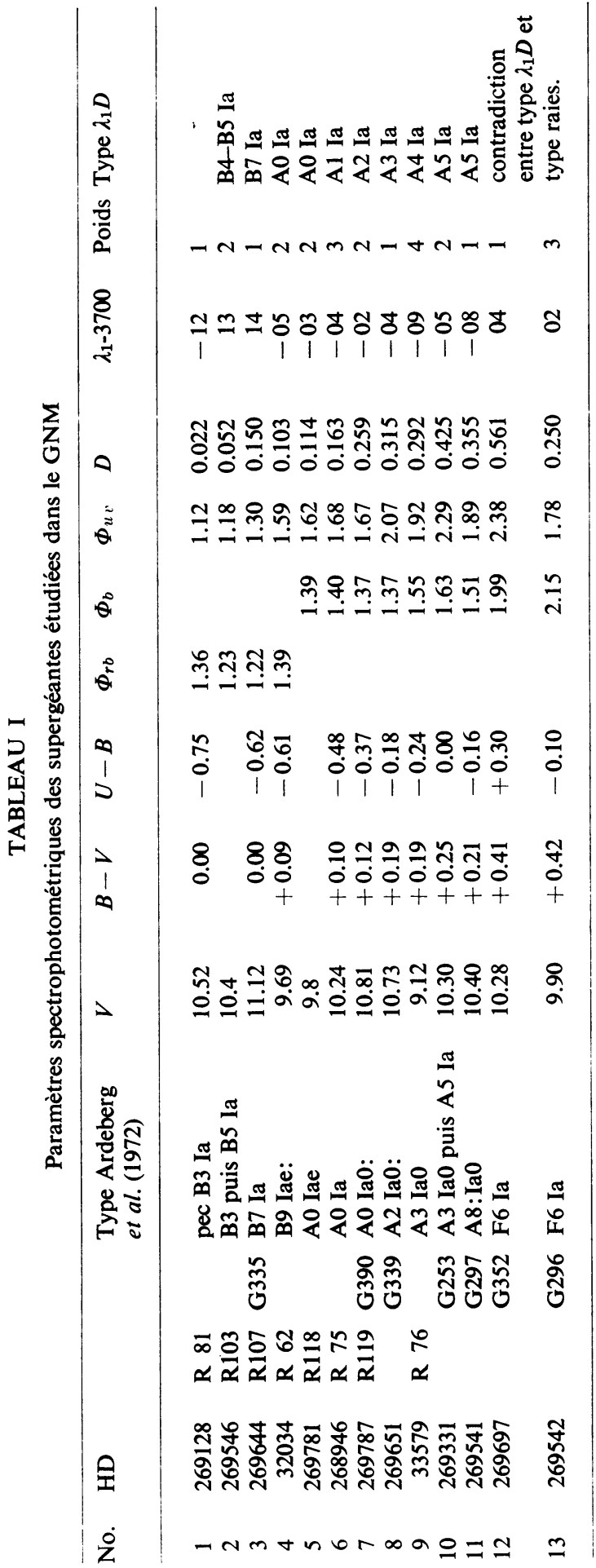




\section{(ii) Classification spectrale}

En extrapolant les courbes d'égal type spectral définies à l'aide des étoiles galactiques ayant des types MKK, on obtient les classification inscrites dans la derniére colonne du Tableau I; elles sont en bon accord avec celles que Prévot et Maurice ont déterminées (Tableau I, colonne 5) et publiées dans Ardeberg et al. (1972).

\section{(iii) Corrections d'absorption interstellaire}

Pour les étoiles du GNM, l'absorption interstellaire est faible et la forme de la loi absorption adoptée pour la corriger importe peu; nous avons adopté une absorption normale pour laquelle $A_{v}=1.9 e_{b}$ (ou $1.7 e_{r b}$ ).

Le calcul des excès de gradient a été fait comme dans le cas des étoiles de $\mathrm{h}$ et $\chi$ Per qui ont servi à calibrer en magnitudes absolues la partie du diagramme $\lambda_{1} D$ la plus proche de celle des étoiles du GNM étudiées ici: on a adopté la valeur de $\Phi_{o b}$ (égale à $\Phi_{\text {orb }}$ pour les étoiles B) lue par interpolation sur les courbes d'égal type spectral (ou sur leur extrapolation) dans le diagramme $\lambda_{1} D$. Ces valeurs sont données par les colonnes 7 et 10 du Tableau II; les colonnes 8 et 11 donnent les valeurs de $A_{v}$ en magnitudes et la colonne 12 la valeur $V_{o}$ de la magnitude $V$ après correction de l'absorption interstellaire.

On peut remarquer que la valeur moyenne des $A_{v}$ que nous obtenons $(0.6 \mathrm{mag}$. environ) est un peu supérieure à la valeur moyenne généralement admise $(0.3 \mathrm{mag}$. $)$

\section{(iv) Module de distance du GNM}

Parmi les étoiles du Tableau II, certaines se situent dans la partie du diagramme $\lambda_{1} D$ déjà calibrée en magnitudes absolues et on peut lire les valeurs de $M$ correspondantes sur la Figure 1 (Tableau II, colonne 13) puis faire le calcul du module de distance, $V_{o}-M_{l u}$, du GNM (Tableau II, colonne 14); les 4 valeurs obtenues se situent entre 17,8 et 18,4 avec une moyenne de 18,1 . Cette valeur plus faible que celles généralement admises, est tout à fait provisoire car le nombre d'observations est encore insuffisant. L'intérêt de la méthode est que le résultat est indépendant des étoiles RR Lyr et des Céphéïdes.

On peut remarquer également que les hypothèses faites sur les couleurs intrinsèques des supergéantes du GNM entre $M=-7$ et $M=-8$, hypothèses qui entraînent une valeur un peu supérieure à celle généralement admise pour l'absorption interstellaire, sont sans influence sur la valeur obtenue ici pour le module de distance; en effet les mêmes hypothèses ont été faites pour le calcul des magnitudes absolues des étoiles galactiques situées dans la même région du diagramme $\lambda_{1} D$ et les changer reviendrait à changer de la même quantité les valeurs de $M$ pour les étoiles de la galaxie et pour le GNM; les courbes d'égale magnitude absolue se déplaceraient un peu dans le plan $\lambda_{1} D$ mais la valeur du module de distance du GNM resterait le même.

Par contre toute modification du module de distance que nous avons admis pour $\mathrm{h}$ et $\chi$ Per (11.8) entraînerait une modification correspondante de la distance du GNM et on peut dire que la détermination faite ici est celle de la différence entre les modules de distance de h et $\chi$ Per et du GNM. 


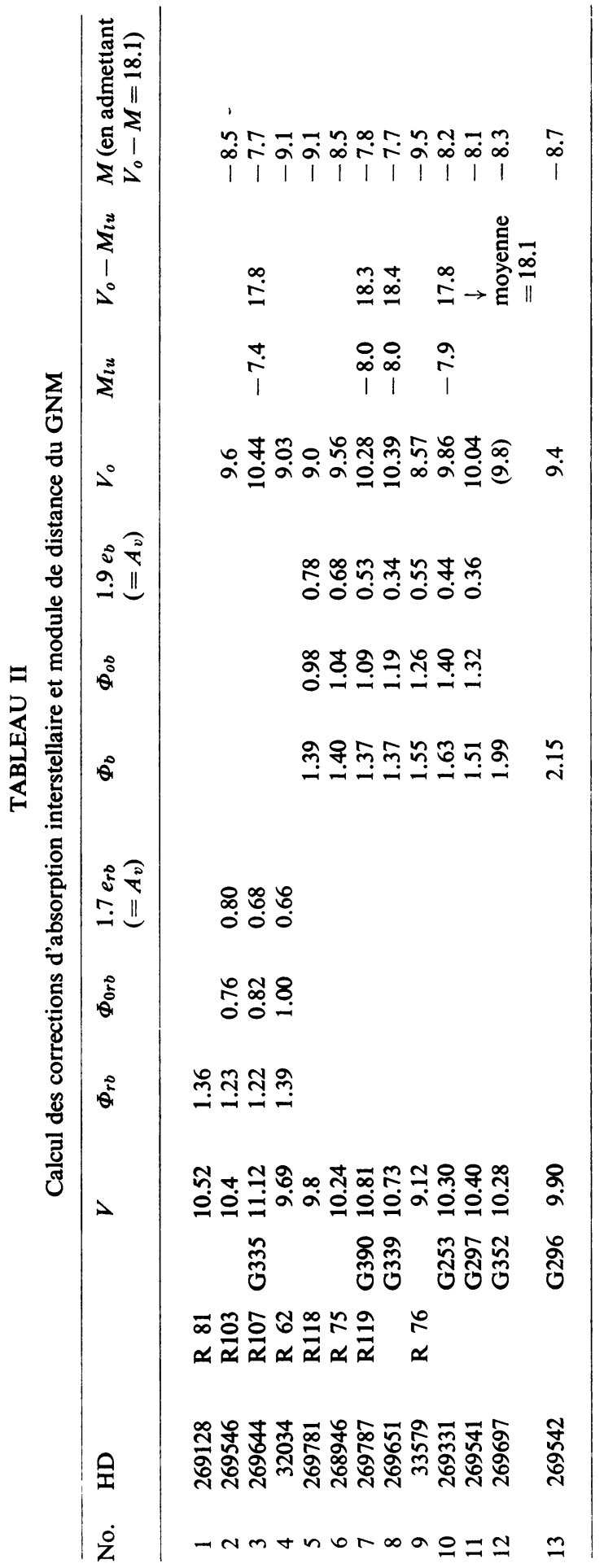


(v) Extension de la calibration du plan $\lambda_{1} D$ en magnitudes absolues

Dans la dernière colonne du Tableau II on a inscrit pour chaque étoile la valeur de la magnitude absolue en admettant 18.1 comme module de distance du GNM. On voit facilement, en reportant ces magnitudes absolues sur la Figure 1, que les étoiles plus brillantes que $M=-8$ (à la seule exception de $\mathrm{G} 253$ ) sont bien situées au delà de la courbe $M=-8$ et que l'on pourrait tracer une nouvelle courbe d'égale magnitude $M=-9$ nettement séparée de la courbe $M=-8$.

\section{(vi) Cas des étoiles F6 Ia}

Nous avions inclus dans le programme d'observation G 352 et G 296 classées toutes deux F6 Ia par Prévot et Maurice, car leurs positions dans le diagramme $U B V$ différaient notablement, sans que l'on puisse dire laquelle était anormale. Les spectres obtenus par nous donnent bien, en ce qui concerne les raies, un type F6 Ia et les deux étoiles ont des spectres très analogues dans le domaine 6200-4000 $\AA$. Par contre leurs discontinuités de Balmer différent considérablement: 0.561 et 0.250 ; il en résulte que l'une d'elles se classe F0 Ia en $\lambda_{1} D$ et l'autre G0 Ia; elles sont donc toutes les deux anormales. Ce phénomène qui n'a jamais été observé pour les étoiles F6 Ia de la galaxie est probablement fréquent dans le GNM car plusieurs étoiles de ces types se placent en $U B V$ entre les position extrêmes occupées par G 352 et G 296. Aucune explication satisfaisante n'a être trouvée.

\section{Bibliographie}

Ardeberg, A., Brunet, J. P., Maurice, E., et Prévot, L.: 1972, Europ. Southern Obs., preprint No. 1. Baillet, A., Chalonge, D., et Cojan, J.: 1952, Ann. Astrophys. 15, 144.

Chalonge, D.: 1958, Rich. Astron. 5, 345.

Chalonge, D.: 1966, in K. Lodén, L. O. Lodén and U. Sinnerstad (eds.), 'Spectral Classification and Multicolour Photometry', IAU Symp. 24, 77.

Chalonge, D. et Divan, L.: 1952, Ann. Astrophys. 15, 201.

Chalonge, D. et Divan, L.: 1973, Astron. Astrophys. 23, 69.

Chalonge, D. et Servigne, M.: 1952, Ann. Astrophys. 15, 151.

Laffineur, M.: 1952, Ann. Astrophys. 15, 154.

\section{DISCUSSION}

Van den Bergh: Could your indices be affected by small differences in composition between the super giants in the Galaxy and the Large Cloud?

Divan: Our indices depend only on the continuum of the stars and are very little affected by variations in chemical composition for the region of the $\lambda_{1} D$ diagram in which those $B$ and early $A$ supergiants are located. Moreover, the few chemical analysis of LMC stars done until now have revealed no really significant differences between LMC stars and the Sun.

Jaschek: In the last years it has become evident that even among the early B-type supergiants there are peculiarities like the $\mathrm{N}$ anomaly discussed by Walborn and the Jaschek's and the $\mathrm{O}$ anomaly found by the Jaschek's. It is therefore important to watch for possible difference in the $M_{v}$ 's, before lumping together the $M$ coming from different groups. 